Original Article (short paper)

\title{
Effect of linear and undulating training periodization models on the repeated sprint ability and strength of soccer players
}

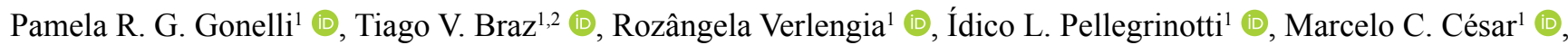

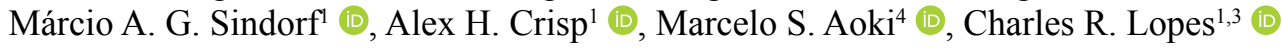 \\ ${ }^{1}$ Universidade Metodista de Piracicaba, UNIMEP, Human Performance Research Laboratory, SP, Piracicaba, \\ Brazil; ${ }^{2}$ Faculdade de Americana, Americana, SP, Brazil; ${ }^{3}$ Faculdade Adventista de Hortolandia, Hortolândia, SP, \\ Brazil; ${ }^{4}$ Universidade de São Paulo, USP, School of Arts, Sciences and Humanities, São Paulo, SP, Brazil.
}

\begin{abstract}
Purpose: The aim of the study was to investigate the effect of linear and undulating strength-power training scheme on the repeated sprint ability (RSA) and lower body strength of soccer players. Method: Twenty soccer players (under- 20 category) were split into 2 groups: the linear load ( $L L, n=10)$ and the undulating load (UL, $n=10)$. In the commencement and at the end of the 6-week pre-season period, the RSA test $(6 \times 35 \mathrm{~m})$ and the 1RM parallel squat test $\left(1 \mathrm{RM}_{\text {squat }}\right)$ were conducted. The LL and UL performed the same type and number of sessions. The training stimulus in the strength training was different between LL (Weeks 1 and $2=$ Muscular Endurance; Weeks 3 and $4=$ Strength; Weeks 5 and $6=$ Power) and UL (daily load variation in the same week). Results: A improvement in RSA and $1 \mathrm{RM}_{\text {squat }}$ was detected in LL and in UL. No significant difference was noted between LL vs UL for all variables. Conclusion: Both groups improved maximal muscle strength in parallel back squat and RSA. UL induced a greater gain in RSA.
\end{abstract}

Keywords: soccer; strength; speed; athletic training.

\section{Introduction}

The repeated-sprint ability (RSA) is the ability to produce the best possible average sprint performance over a series of sprints ( $\leq 10$ seconds), separated by short ( $\leq 60$ seconds) recovery periods ${ }^{1}$. This ability is a complex quality to be related to both neuromuscular (determining maximal sprint speed, e. g., neural drive, motor unit activation or muscle strength) and metabolic sprint ability (involved in the ability to repeated sprints, e. g., oxidative capacity for phosphocreatine $(\mathrm{PCr})$ recovery, lactate- $\mathrm{H}^{+}$ buffering or $\mathrm{Na}^{+} / \mathrm{K}^{+}$transport capacity $)^{2,3}$. It also discriminates against professional soccer players from amateurs ${ }^{4}$. In addition, stronger lower body soccer players have better performance during sprints, change of direction and vertical jump ${ }^{5}$. Strength is relevant to soccer players; therefore, it is recommended including different training strategies to improve their physical attributes ${ }^{6}$. Organizing soccer training sessions is especially challenging because it requires many fitness components [e.g., aerobic capacity, RSA, maximal muscle strength and (or) power strength] essential for elite soccer players?

Most popular strength-power training schemes are the linear (LL) and the undulating linear strength-power training schemes $(\mathrm{UL})^{8}$. In LL the initial cycle volume is high and the intensity is low and, as the training progresses through specific cycles, the training volume decreases, whereas the training intensity gradually increases ${ }^{9}$. Therefore, the second cycle is featured by moderate intensity and volume, and by a marked increment in intensity and a concomitant decrement in volume, in the last cycle ${ }^{8}$. On the other hand, the UL relies on the non-linear manipulation of volume and intensity throughout the training cycle ${ }^{10}$. Such training models combine short, high-volume and short, high-intensity training periods which are alternated by 1 -week intervals ${ }^{8}$.

Fleck and Kraemer ${ }^{8}$ suggested the use of UL strength-power training scheme in team sports who must have a higher performance level (muscular strength, power, endurance, and aerobic capacity) throughout the entire course of the competitive season. These authors have stated that the low performance at the beginning of the season and the risk of fatigue accumulation due to high training volumes are the disadvantages of LL strength-power scheme training. Moreover, most studies about strength training (ST) in the soccer modality are associated with the exercises (i.e., full squat vs. plyometric exercises) ${ }^{11}$, the training method (i.e., traditional strength training vs complex contrast training $)^{10}$, and the combination with other training sessions or with the period of the season. Few studies have assessed the effect of LL and UL strengthpower scheme training approaches on athletic populations $s^{13,14}$, especially on soccer players. In addition, there is limited research about the LL and UL strength-power scheme training, especially concerning volume and intensity in soccer players ${ }^{15}$.

The present study has compared the chronic effect of 2 distinct strength-power training schemes (LL and UL), with equated volume and intensity on the RSA and 1RM parallel squat performed by elite young Brazilian soccer players (Under 20) from the same soccer club. Based on the concept by Fleck and $\mathrm{Kraemer}^{8}$, the initial hypothesis of the present study states that the UL would induce superior training outcomes (RSA and $1 \mathrm{RM}_{\text {squat }}$ ) than the LL strength-power training schemes. As far as it is known by the authors, the present research is the first one 
to compare the chronic effects of the LL and UL strength-power training schemes on young elite soccer players from the same club.

\section{Methods}

The present study followed a stratified-randomized longitudinal design involving 20 young male elite Brazilian soccer players. Participants were pair-matched according to baseline strength $\left(1 \mathrm{RM}_{\text {squat }}\right)$ and then randomly assigned to $(\mathrm{LL}, \mathrm{n}=10)$ and (UL, $\mathrm{n}=10)$ groups (table 1). The subjects were selected in a soccer team (under-20 category) participating in the category's national championship. The study was conducted in the beginning and at the end of the 6-week pre-season training. Goalkeepers were excluded from the experiment. Each participant visited the laboratory 2 times, in a 24-hour interval. All subjects were already familiar with the tests applied. The preliminary session was focused on explaining and recommending the experimental protocols. The subjects underwent anthropometry and body composition measurements, as well as maximum cardiorespiratory tests on the treadmill. The second session consisted of assessing the $1 \mathrm{RM}_{\text {squat }}$ and the RSA through the running anaerobic sprint test (RAST). The subjects attended 30 training sessions and the tests were performed before and after the herein proposed intervention.

\section{Subjects}

Twenty male athletes (under-20 category) have volunteered to participate in the present study (Table 1). All participants belonged to a soccer team participating in Brazilian national tournaments (1st division). The inclusion criteria were: (a) having at least 4 years of experience in soccer; (b) having at least 1-year experience in resistance training; (c) attending all training sessions and matches; (d) not having any injury able to interfere in the study; (e) not being away from training for over a month prior to the beginning of the study. Parents or guardians have signed the under-age athletes' consent form. The present study was approved by the local Research Ethics Committee (protocol n. 16/14).

\section{Procedures}

Training Periodization. The participants attended 3 strength-training sessions per week (Mondays, Wednesdays, and Fridays), besides the normal training session with the whole team, for 6 consecutive weeks, during the pre-season (November/December). The 18 strength-training (ST) sessions were divided into 6 strength endurance (SE), 6 maximum strength (MS) and 6 power strength (PS) sessions. The MS training sessions were conducted in the morning $(\mathrm{M}-8.30$ $10.30 \mathrm{am})$, and the others, in the afternoon (A - 14.30-16.30 pm). The endurance training consisted of 3 sets of $15 \mathrm{RM}$ repetitions, with 60 -second intervals between them, and 2-minute intervals between exercises such as the back squat, leg press, leg curls, calf raise, bench press, seated cable row, frontal bar lifting, and upper and dorsal abdominals. The maximal strength training comprised 3 sets of 3RM, 2 to 4 minutes between the series and the squatting, leg curl, bench press, paddling and leg press exercises. The power strength training consisted of 4-6 series of 8 continuous jumps over 60 -cm obstacles and of 5 -meter sprints. Subsequently, the athletes performed other 5-8 series of 8 drop jumps with $60-\mathrm{cm}$ fall height and 2 to 3 -minute intervals between the series and the exercises.

The conventional training consisted of 6 sessions of Small Sided Games (SSG), 24 tactical technical training sessions (TT) and 12 Repeated Sprint Ability Trainings (RSA). The SSG was performed with 8 sets of 4 minutes, 3 vs. 3 soccer players (without the goalkeeper) in a 25-m width and 18-m long field. The RSA was split into 2 types of the training session ( $\mathrm{RSA}^{1}$; $\left.\mathrm{RSA}^{2}\right)$. The RSA ${ }^{1}$ consisted of 5 series of $5(40 \mathrm{~m})$ sprints, with 20 -second intervals between repetitions and 10-minute intervals between series. The $\mathrm{RSA}^{2}$ was performed in 1 series of $15(10 \mathrm{~m})$ sprints with direction change every 15 meters, a 1-minute interval between series and 8-minute intervals between exercises. The SSG and RSA sessions were conducted in the afternoon. The TTs were performed in the morning. All training sessions began with standardized warm-up exercises for approximately 10 minutes (running drills and stretching exercises). Over the course of each training week, all subjects performed the same exercises and repetition volume in sessions SSG, TT, and RSA throughout the duration of the study (Table 2).

Subjects in the LL group were trained using the linear load distribution in the ST sessions (Weeks 1 and $2=$ Strength Endurance; Weeks 3 and 4 = Maximum Strength; Weeks 5 and 6 = Power). The UL group underwent the same ST sessions; however, using daily load variations within the same training week (Weeks 1 to $6=$ Strength Endurance, Maximum Strength and Power). Both groups (LL and UL) performed the same type and attended the same number of sessions; however, the load distribution in the ST was different (figure 1).

Table 1. Sampling of descriptive features (mean \pm SD).*

\begin{tabular}{|c|c|c|c|c|c|c|c|c|}
\hline Groups & $\begin{array}{c}\text { Age } \\
\text { (years) }\end{array}$ & $\begin{array}{l}\text { Height } \\
\text { (m) }\end{array}$ & $\begin{array}{c}\text { Body Mass } \\
(\mathrm{Kg})\end{array}$ & $\%$ Fat & $\begin{array}{c}\text { VT } \\
(\mathrm{km} / \mathrm{h})\end{array}$ & $\begin{array}{c}\mathrm{RCP} \\
(\mathrm{km} / \mathrm{h})\end{array}$ & $\begin{array}{c}\mathrm{VO}_{2 \max } \\
\left(\mathrm{mL} \cdot \mathrm{min}^{-1} \cdot \mathbf{k g}^{-1}\right)\end{array}$ & $\begin{array}{c}\text { 1RM }_{\text {squat }} \\
(\mathrm{kg})\end{array}$ \\
\hline $\operatorname{LL}(\mathrm{n}=10)$ & $18.1 \pm 0.7$ & $1.82 \pm 0.1$ & $74.9 \pm 6.9$ & $10.2 \pm 2.0$ & $11.1 \pm 1.1$ & $13.4 \pm 1.0$ & $63.3 \pm 5.2$ & $145 \pm 35$ \\
\hline $\mathrm{UL}(\mathrm{n}=10)$ & $18.3 \pm 0.9$ & $1.80 \pm 0.1$ & $75.9 \pm 9.4$ & $10.9 \pm 2.3$ & $11.2 \pm 0.6$ & $13.4 \pm 0.8$ & $62.0 \pm 4.6$ & $153 \pm 16$ \\
\hline
\end{tabular}

$\mathrm{LL}=$ linear load group; $\mathrm{UL}=$ undulating load group; $\% \mathrm{Fat}=$ fat rate; $\mathrm{VT}=$ ventilatory threshold; $\mathrm{RCP}=$ respiratory compensation point; $\mathrm{VO}{ }_{2 \max }=$ maximal oxygen uptake; $1 \mathrm{RM}_{\text {squat }}=$ maximal muscle strength in parallel squat test; $*=$ no significant differences were found between the LL and UL groups ( $\mathrm{p}>0.05)$. 
Table 2. Distribution of training sessions for the Linear Load and Undulating Load groups.

\begin{tabular}{|c|c|c|c|c|c|c|}
\hline & Period & Monday & Tuesday & Wednesday & Thursday & Friday \\
\hline \multicolumn{7}{|c|}{ Linear Load distribution of the strength training (LL) } \\
\hline \multirow{2}{*}{ Week 1} & M & TT & TT & $\mathrm{TT}$ & TT & TT \\
\hline & A & $\operatorname{SE}\left(3^{15}\right)$ & SSG & $\operatorname{SE}\left(3^{15}\right)$ & $\mathrm{RSA}^{1}$ & $\operatorname{SE}\left(3^{15}\right)$ \\
\hline \multirow{2}{*}{ Week 2} & M & TT & TT & TT & TT & TT \\
\hline & A & $\operatorname{SE}\left(3^{15}\right)$ & SSG & $\operatorname{SE}\left(3^{15}\right)$ & $\mathrm{RSA}^{1}$ & $\operatorname{SE}\left(3^{15}\right)$ \\
\hline \multirow{2}{*}{ Week 3} & M & $\operatorname{MS}\left(3^{4}\right)$ & $\mathrm{TT}$ & $\operatorname{MS}\left(3^{4}\right)$ & TT & $\operatorname{MS}\left(3^{4}\right)$ \\
\hline & A & $\mathrm{RS}^{\mathrm{A}} 2$ & SSG & $\mathrm{RSA}^{2}$ & RSA $^{1}$ & $\mathrm{RSA}^{2}$ \\
\hline \multirow{2}{*}{ Week 4} & M & $\operatorname{MS}\left(3^{4}\right)$ & $\mathrm{TT}$ & $\operatorname{MS}\left(3^{4}\right)$ & $\mathrm{TT}$ & $\operatorname{MS}\left(3^{4}\right)$ \\
\hline & A & $\mathrm{RSA}^{2}$ & SSG & $\mathrm{RSA}^{2}$ & $\mathrm{RSA}^{1}$ & $\mathrm{RSA}^{2}$ \\
\hline \multirow{2}{*}{ Week 5} & M & TT & $\mathrm{TT}$ & $\mathrm{TT}$ & TT & $\mathrm{TT}$ \\
\hline & A & $\operatorname{PS}\left(4^{8 \mathrm{HJ}}+5^{8 \mathrm{DJ}}\right)$ & SSG & $\operatorname{PS}\left(4^{8 \mathrm{HJ}}+5^{8 \mathrm{DJ}}\right)$ & $\mathrm{RSA}^{1}$ & $\operatorname{PS}\left(4^{8 \mathrm{HJ}}+5^{8 \mathrm{DJ}}\right)$ \\
\hline \multirow{2}{*}{ Week 6} & M & $\mathrm{TT}$ & $\mathrm{TT}$ & $\mathrm{TT}$ & $\mathrm{TT}$ & $\mathrm{TT}$ \\
\hline & A & PS $\left(6^{8 \mathrm{HJ}}+8^{8 \mathrm{DJ}}\right)$ & SSG & PS $\left(6^{8 \mathrm{HJ}}+8^{8 \mathrm{DJ}}\right)$ & RSA $^{1}$ & $\operatorname{PS}\left(6^{8 \mathrm{HJ}}+8^{8 \mathrm{DJ}}\right)$ \\
\hline \multicolumn{7}{|c|}{ Daily Undulating Load distribution of the strength training (UL) } \\
\hline \multirow{2}{*}{ Week 1} & $\mathrm{M}$ & MS $\left(3^{4}\right)$ & TT & TT & TT & TT \\
\hline & $\mathrm{A}$ & $\mathrm{RSA}^{2}$ & SSG & $\mathrm{PS}\left(4^{8 \mathrm{HJ}}+5^{8 \mathrm{DJ}}\right)$ & $\mathrm{RSA}^{1}$ & $\operatorname{SE}\left(3^{15}\right)$ \\
\hline \multirow{2}{*}{ Week 2} & M & $\operatorname{MS}\left(3^{4}\right)$ & $\mathrm{TT}$ & $\mathrm{TT}$ & $\mathrm{TT}$ & $\mathrm{TT}$ \\
\hline & A & $\mathrm{RSA}^{2}$ & SSG & $\mathrm{PS}\left(4^{8 \mathrm{HJ}}+5^{8 \mathrm{DJ}}\right)$ & $\mathrm{RSA}^{1}$ & $\operatorname{SE}\left(3^{15}\right)$ \\
\hline \multirow{2}{*}{ Week 3} & M & $\operatorname{MS}\left(3^{4}\right)$ & $\mathrm{TT}$ & $\mathrm{TT}$ & $\mathrm{TT}$ & TT \\
\hline & A & $\mathrm{RSA}^{2}$ & SSG & $\mathrm{PS}\left(4^{8 \mathrm{HJ}}+5^{8 \mathrm{DJ}}\right)$ & $\mathrm{RSA}^{1}$ & $\operatorname{SE}\left(3^{15}\right)$ \\
\hline \multirow{2}{*}{ Week 4} & M & $\operatorname{MS}\left(3^{4}\right)$ & $\mathrm{TT}$ & $\mathrm{TT}$ & TT & TT \\
\hline & A & $\mathrm{RSA}^{2}$ & SSG & PS $\left(6^{8 \mathrm{HJ}}+8^{8 \mathrm{DJ}}\right)$ & $\mathrm{RSA}^{1}$ & $\operatorname{SE}\left(3^{15}\right)$ \\
\hline \multirow{2}{*}{ Week 5} & M & $\operatorname{MS}\left(3^{4}\right)$ & $\mathrm{TT}$ & $\mathrm{TT}$ & $\mathrm{TT}$ & TT \\
\hline & A & $\mathrm{RSA}^{2}$ & SSG & $\operatorname{PS}\left(6^{8 \mathrm{HJ}}+8^{8 \mathrm{DJ}}\right)$ & $\mathrm{RSA}^{1}$ & $\operatorname{SE}\left(3^{15}\right)$ \\
\hline \multirow{2}{*}{ Week 6} & M & $\operatorname{MS}\left(3^{4}\right)$ & $\mathrm{TT}$ & TT & TT & TT \\
\hline & A & $\mathrm{RSA}^{2}$ & SSG & $\operatorname{PS}\left(6^{8 \mathrm{HJ}}+8^{8 \mathrm{DJ}}\right)$ & $\mathrm{RSA}^{1}$ & $\operatorname{SE}\left(3^{15}\right)$ \\
\hline
\end{tabular}

$\mathrm{LL}=$ Linear Load Group; UL= Undulating Load Group; $\mathrm{M}=$ Morning (8.30-10.30 am); A= Afternoon (14.30-16.30 pm); MS= Maximal Strength; PS= Power Strength Training; SE = Strength Endurance; RSA= Repeated Sprint Ability Training; SSG= Small Sided Games Training; TT= Tactical Technical Training; $3^{15}, 3^{4}$ and $6^{8}=$ Numbers 3-6 represent the number of sets, whereas the numbers 4-8-15 describe the number of repetitions; HJ = Horizontal Jump; DJ = Drop Jump.

Figure 1. Mean weekly (W) values for number of strength training repetitions in linear load (LL) and undulating load (UL) during the study.

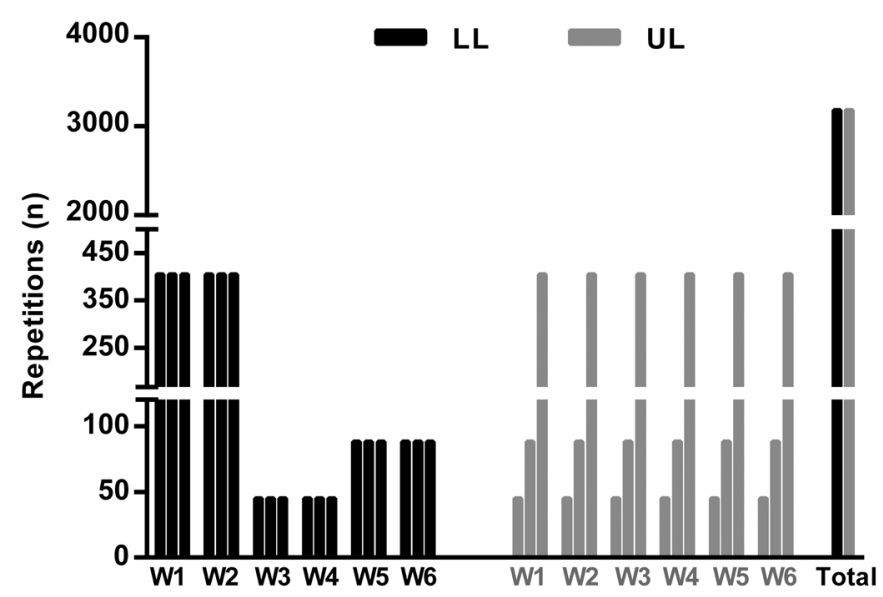


Tests. The athletes rested the day before the evaluations and were instructed to keep the regular dietary intake (food and beverages), as well as to avoid having stimulating drinks (e.g. caffeinated drinks) and alcohol consumption 24 hours before the adopted procedures. One year before to research, all subjects had already performed the tests (RSA, 1RM ${ }_{\text {squat }}$ ) on at least 5 occasions.

Repeated sprint ability test (RSA). The RSA test was performed on a natural grass surface in the team's field. It consisted of $6(35 \mathrm{~m})$ straight shuttle sprints. The participants started a newsprint every 10 seconds of passive recovery. An electronic photocell system was used to record the time of the sprints (Speed Test 6.0 CEFISE $®$, Nova Odessa, SP, Brazil). The best sprint time $\left(\mathrm{RSA}_{\text {best }}\right)$, the mean sprint time $\left(\mathrm{RSA}_{\text {mean }}\right)$ and the sprint decrease rate $\left(\mathrm{RSA}_{\text {index }}\right)$ were recorded for statistical

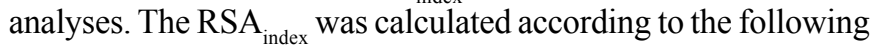
equation: $\left[\left(\Sigma 6\right.\right.$ times / $\mathrm{RSA}_{\text {best }}$ x 6$\left.\left.)-1\right] * 100\right)$; wherein $\Sigma 6$ times is the time spent to complete the $6 \times 35 \mathrm{~m}$ shuttle sprints ${ }^{3}$.

The Maximal Muscle Strength in parallel squat exercises $\left(1 \mathrm{RM}_{\text {squat }}\right)$. The maximal muscle strength in the parallel squat exercise (Smith machine) was assessed through the $1 \mathrm{RM}$ test ${ }^{16}$. Briefly, the subjects performed 2-3 sets of warm-ups with 5-10 repetitions at approximately $40 \%$ to $60 \%$ of their estimated 1 $\mathrm{RMs}$, before the protocol. The subjects were asked to perform squatting exercises until reaching up to $90^{\circ}$ knee-angle (tightly controlled). The test was performed with at most 5 (3-5 minutes) rest intervals between each attempt.

\section{Statistical analyses}

The variance normality and homogeneity were assessed through the Shapiro-Wilk and Levene tests, respectively. The paired t-test was used to check the RSA and $1 \mathrm{RM}_{\text {squat }}$ differences from pre to post training. The adopted significance was $\mathrm{p} \leq 0.05$. Analyses were conducted in SPSS-22.0 software (IBM Corp.,
Armonk, NY, USA). The practical significance was assessed by calculating the Cohen's d effect size (ES). The equation 1 for time [(post-test score - pre-test score)/pooled pre-test and post-test standard deviation)] and 2 for comparing groups [(mean change group 1 - mean change group 2) / pooled standard deviation of the change score] were used. The $\mathrm{ES}<0.2$, $0.2-0.6,0.6-1.2,1.2-2.0$ and $2.0-4.0$ were considered trivial, small, moderate, large and very large, respectively ${ }^{17}$. If the $90 \%$ confidence limits overlapped, small positive and negative values for the magnitude were deemed unclear; otherwise, that magnitude was deemed to be the observed magnitude ${ }^{17}$. Data analysis was performed using a modified statistical Excel spreadsheet ${ }^{17}$.

\section{Results}

No difference was observed between pre vs post-test in the $\mathrm{RSA}_{\text {best }}$ in $\mathrm{LL}(\mathrm{p}=0.692 ; \mathrm{ES}=-0.16,90 \% \mathrm{CI}=0.72)$ and $\mathrm{UL}$ $(\mathrm{p}=0.204 ; \mathrm{ES}=-0.53,90 \% \mathrm{CI}=0.71)$. There was improvement in $\mathrm{RSA}_{\text {mean }}$ in the LL group $(\mathrm{p}=0.028 ; \mathrm{ES}=-1.10,90 \% \mathrm{CI}=0.76)$ and in the UL group ( $\mathrm{p}=0.001 ; \mathrm{ES}=-1.90,90 \% \mathrm{CI}=0.53$ ) when compared to the pre-intervention scores. The RSA index $_{\text {was }}$ improved in the UL group $(\mathrm{p}=0.024 ; \mathrm{ES}=-0.98,90 \% \mathrm{CI}=0.66)$, but not LL $(\mathrm{p}=0.089 ; \mathrm{ES}=-1.00,90 \% \mathrm{CI}=0.97)$. However, similar ES and relative improvement (\%) were found in LL and UL (-25.4\% vs $-24.0 \%$; ES $=-1.00$ vs -0.98$)$, respectively. A significant increase in the $1 \mathrm{RM}_{\text {squat }}$ was observed in the LL group $(\mathrm{p}=0.023 ; \mathrm{ES}=0.73,90 \% \mathrm{CI}=0.49)$ and in the $\mathrm{UL}$ group $(\mathrm{p}=0.004 ; \mathrm{ES}=0.82,90 \% \mathrm{CI}=0.39)$ after the training intervention (Table 3 ).

There was no significant difference noted between LL vs UL in $\mathrm{RSA}_{\text {best }}(\mathrm{d}=0.32 ; 90 \% \mathrm{CI}=0.78$; small unclear $\mathrm{ES} ; \mathrm{p}=0.48)$, $\operatorname{RSA}_{\text {mean }}(\mathrm{d}=0.38 ; 90 \% \mathrm{CI}=0.82 ;$ small unclear ES; $\mathrm{p}=0.41)$, $\mathrm{RSA}_{\text {index }}(\mathrm{d}=0.01 ; 90 \% \mathrm{CI}=0.35$; trivial $\mathrm{ES} ; \mathrm{p}=0.98)$ and $1 \mathrm{RM}$ squa $(d=0.20 ; 90 \% C I=0.80$; trivial $E S ; p=0.66)$ (figure 2$)$.

Table 3. Maximal strength and repeated sprint ability results before and after the linear (LL) and undulating loads (UL).

\begin{tabular}{|c|c|c|c|c|c|c|c|c|c|}
\hline & Variables & $\mathbf{L L}(\mathbf{n}=10)$ & $\Delta \%$ & $\mathbf{p}$ & $\begin{array}{c}\text { ES }( \pm 90 \% \text { CL) } \\
\text { classification }\end{array}$ & UL (n=10) & $\Delta \%$ & $\mathbf{p}$ & $\begin{array}{c}\mathbf{E S}(\mathbf{( 9 0 \%} \mathbf{C L}) \\
\text { classification }\end{array}$ \\
\hline \multirow[t]{4}{*}{ Pre } & $1 \mathrm{RM}_{\text {squat }}(\mathrm{kg})$ & $145 \pm 35$ & & & & $153 \pm 16$ & & & \\
\hline & $\mathrm{RSA}_{\text {best }}(\mathrm{s})$ & $4.70 \pm 0.27$ & & & & $4.61 \pm 0.24$ & & & \\
\hline & $\mathrm{RSA}_{\text {mean }}(\mathrm{s})$ & $5.18 \pm 0.26$ & & & & $5.10 \pm 0.12$ & & & \\
\hline & $\mathrm{RSA}_{\text {index }}(\%)$ & $10.5 \pm 5.8$ & & & & $10.9 \pm 5.8$ & & & \\
\hline \multirow[t]{4}{*}{ Post } & $1 \mathrm{RM}_{\text {squat }}(\mathrm{kg})$ & $166 \pm 23^{*}$ & 18.2 & 0.023 & $\begin{array}{c}0.73(0.49) \\
\text { moderate }\end{array}$ & $170 \pm 26^{*}$ & 10.8 & 0.004 & $\begin{array}{l}0.82(0.39) \\
\text { moderate }\end{array}$ \\
\hline & $\mathrm{RSA}_{\text {best }}(\mathrm{s})$ & $4.66 \pm 0.10$ & -0.4 & 0.692 & $\begin{array}{c}-0.16(0.72) \\
\text { trivial }\end{array}$ & $4.50 \pm 0.15$ & -2.2 & 0.204 & $\begin{array}{c}-0.53(0.71) \\
\text { small }\end{array}$ \\
\hline & $\mathrm{RSA}_{\text {mean }}(\mathrm{s})$ & $4.96 \pm 0.11^{*}$ & -3.9 & 0.028 & $\begin{array}{c}-1.10(0.76) \\
\text { moderate }\end{array}$ & $4.81 \pm 0.17^{*}$ & -5.8 & $<0.001$ & $\begin{array}{c}-1.90(0.53) \\
\text { large }\end{array}$ \\
\hline & $\mathrm{RSA}_{\text {index }}(\%)$ & $6.5 \pm 1.8$ & -25.4 & 0.089 & $\begin{array}{c}-1.00(0.97) \\
\text { moderate }\end{array}$ & $6.8 \pm 2.1^{*}$ & -24.0 & 0.024 & $\begin{array}{c}-0.98(0.66) \\
\text { moderate }\end{array}$ \\
\hline
\end{tabular}

$\mathrm{LL}=$ linear load group; $\mathrm{UL}=$ undulating load group; $1 \mathrm{RM}_{\text {squat }}=$ maximal muscle strength in parallel squat test; $\mathrm{RSA}_{\text {best }}=$ best sprint time; $\mathrm{RSA}_{\text {mean }}=$ mean sprint time; $\mathrm{RSA}_{\text {index }}=$ percent sprint decrease; $\mathrm{ES}=$ Effect Size; $\mathrm{CL}=$ Confidence Limits. ${ }^{*}$ Significant difference in the comparison of pre intervention $(\mathrm{P}<0.05)$. 
Figure 2. Differences in maximum strength in parallel back squat $\left(1 \mathrm{RM}_{\text {squat }}\right)$, sprint decrease rate $\left(\mathrm{RSA}_{\text {index }}\right)$, best sprint time $\left(\mathrm{RSA}_{\text {best }}\right)$ and mean sprint time (RSAmean) between groups linear (LL) and undulating load (UL). Was used Cohen effect-size principle \pm IC $90 \%$ compared absolute differences in raw values of the variables. The grey area represented trivial differences (see methods).

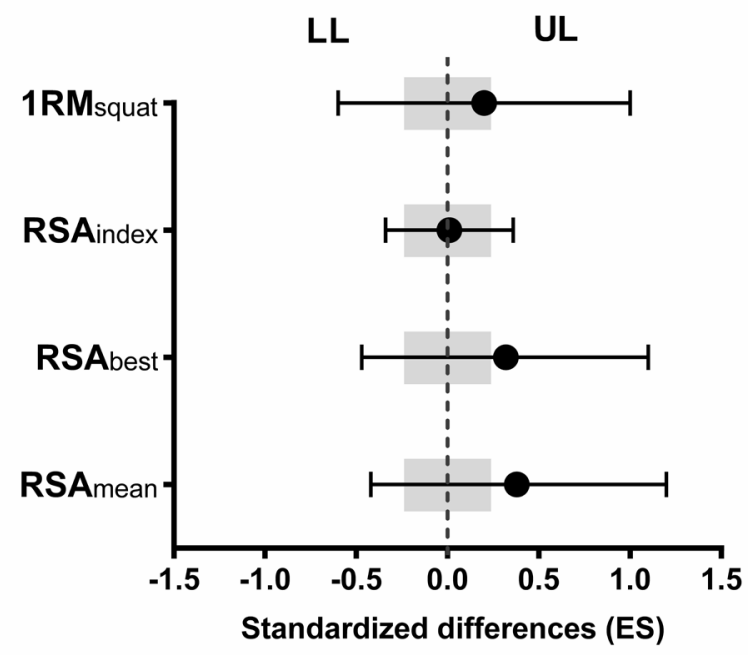

\section{Discussion}

The aim of the present study was to investigate the influence of the LL and UL strength-power training schemes on the RSA and on the lower limb maximal strength of young elite Brazilian soccer players. The major finding in the present study was the significant improvement in the RSA ${ }_{\text {mean }}$ and $1 \mathrm{RM}_{\text {squat }}$, regardless of the implemented strength-power training scheme. The comparison between strength-power training scheme didn't show any difference in variables for LL and UL. All effects sizes comparisons were trivial or small unclear. The present study was the first one to investigate the LL and UL strength-power training schemes throughout the pre-season, in young elite players in the same soccer team (under-20 category).

The 6-week period was enough to improve RSAmean in the LL $(-3.9 \%)$ and UL $(-5.8 \%)$ groups. These rates were higher than the results found by Buccheit, Mendez-Villanueva, Delhomel, Brughelli, Ahmaidi ${ }^{18}\left(-2.5 \% \mathrm{RSA}_{\text {mean }}, 10\right.$-week training) and Tønnessen, Shalfawi, Haugen, Enoksen ${ }^{19}(-2.2 \%$ $\mathrm{RSA}_{\text {mean }}, 10$-week training). It is worth highlighting that these 2 studies did not apply traditional strength exercises, such as parallel squat, leg press and leg curl under high intensity (i.e. 3RM), as it was proposed to the UL and LL groups in the present study. With regard to the strength/power training methods used by soccer players, the high-intensity resistance training (i.e. $>80 \% 1 \mathrm{RM}$ ) seemed to be more efficient than the moderate-intensity resistance training in explosive effort and capacity $\mathrm{RSA}^{20,21}$.

Theoretically, the changes (daily or weekly) in the strength training applied to the UL group could minimize training monotony. There was no improvement in $\mathrm{RSA}_{\text {index }}$ in the UL and LL groups after the 6-week training period (figure 2). Pre and post-intervention comparisons showed similar ES and relative improvement $(\mathrm{LL}$ vs $\mathrm{UL}=-25.4 \%$ vs $-24.0 \%$; ES $=$ $-1.00 \mathrm{vs}-0.98)$. This training stimuli variation for both groups, associated with progressive strength training, is important to the $\mathrm{RSA}_{\text {index }}$ performance, since the effort and recovery patterns in soccer matches can be better described as random (i.e. they are imposed by tactical factors and by the player's ability to self-select the intensity and nature of their efforts) ${ }^{1}$. Indeed, Hill-Haas, Bishop, Dawson, Goodman, Edge ${ }^{21}$ showed an improvement in the RSA of 18 recreationally-active female athletes from different teams after 5 weeks of training (2-5 sets of 15-20RM) with 20-second rest intervals $(+12.5 \%)$ and with 80 -second rest intervals $(+5.4 \%)$. These data showed that the recovery variation and load (work-rest- ratio) in the ST (UL and LL) may improve the RSA index $_{\text {. }}$

On the other hand, no difference was found between the $\mathrm{RSA}_{\text {best }}$ after the training scheme (table 3). Although some studies confirmed the improvement in $\mathrm{RSA}_{\text {best }}$ in 5 -week ${ }^{22}$, 8 -week ${ }^{11}$ and 10 -week ${ }^{18}$ training periods, the improvement in the best sprint performance after the strength and specific training sessions was not entirely confirmed among highly trained soccer players ${ }^{20}$. The impact of strength training on RSA remains unclear ${ }^{2}$. For example, 15 young soccer players who compete in the English Premier League did not present any sprint $(30 \mathrm{~m})$ improvement after 5 weeks of training, even after 10 additional strength-training sessions were provided to the team's traditional training program ${ }^{23}$. A similar result was found by López-Segovia ${ }^{24}$ in their study regarding $30 \mathrm{~m}$ sprints after 16 weeks of training (Team A: $4.25 \mathrm{vs} 4.35 \mathrm{~s}$, ES $=0.58$; Team B: $4.40 \mathrm{vs} 4.36 \mathrm{~s}, \mathrm{ES}=-0.25$ ) applied to 2 soccer teams (under-19 category). According to these researchers ${ }^{24}$, the lack of improvement in the former sprint variables was attributed to the high volume of aerobic exercise, since soccer is a concurrent modality wherein endurance competes with strength/power training ${ }^{20}$. Furthermore, highly trained soccer players need to increase their 1RM half-squat by $23.5 \%$, on average, to achieve approximately $2 \%$ improvement in their best sprint performance at $40-\mathrm{m}$ distances ${ }^{20}$. Such values did 
not meet those observed in the present study $\left(1 \mathrm{RM}_{\text {squat }}=\mathrm{LL}\right.$ $+18.2 \%$, UL $+10.8 \%$ ).

Regardless of the chosen strength-power training scheme, the 6-week pre-season practice period, with 3 weekly sessions, was enough to provide maximal strength $\left(1 \mathrm{RM}_{\text {squat }}\right)$ gains in both the LL $(+18.2 \%)$ and the UL (+10.8\%) groups in the current study. These results corroborate those found by Alvar, Wenner, and Dodd $^{15}$ in their study about collegiate athletes (Division 1), as well as those found by Christou, Smilios, Sotiropoulos, Volaklis, Pilianidis, Tokmakidis ${ }^{25}$, Enright, Morton, Iga, Drust ${ }^{23}$ and Lopes et al. ${ }^{16}$ in their study involving young soccer players. They found values close to those found in the present study in the 6-week cycle (Table 2 ) by applying a LL periodization approach ( 5 weeks, 3 sets of 8 repetitions, $60-80 \%$ of $1 \mathrm{RM} /$, subsequently, another 5 weeks, 4 sets of 6 repetitions and $85 \%$ of $1 \mathrm{RM})$.

Several limitations should be mentioned in the present study. It was not possible implementing a control group to compare the effect of the current interventions. The intervention period (pre-season) was relatively short (6 weeks) and did not examine the maintenance of the training effects on each group during the competitive season. However, due to the nature of the population and to the relatively small sample size (i.e., young elite professional soccer players), it was not possible having multiple experimental training groups or individuals performing isolated strength-training protocols ${ }^{23}$. Examining periodized ST among competitive athletes (e.g. young elite soccer players) is a major challenge since the control groups do not have athletes who avoid ST.

\section{Conclusion}

The current results indicate that strength-power training schemes are equally effective in organizing training sessions in order to improve the physical performance of young elite soccer players. Both the LL and UL models appear to increase the maximal strength in the parallel squat and the mean RSA within the 6-week pre-season. However, there was no improvement in the best sprint of the assessed sample group. It was possible identifying performance improvement in the same variables, in both groups ( $\mathrm{RSA}_{\text {mean }}$ and $1 \mathrm{RM}_{\text {squat }}$ ); and in the RSAindex only in the UL induced a performance increment. Although, the current study pointed out some directions, it is necessary repeating the study using a larger sample and longer duration ( $>6$ weeks) in order to draw more precise conclusions.

\section{References}

1. Bishop D, Girard O, Mendez-Villanueva A. Repeated-sprint ability part II: Recommendations for training. Sport Med. 2011;41(9):741-56.

2. Girard O, Mendez-Villanueva A, Bishop D. Repeated-sprint ability part 1: factors contributing to fatigue. Sport Med. 2011;41(8):673-94.

3. Glaister M, Howatson G, Lockey RA, Abraham CS, Goodwin JE, McInnes G. Familiarization and reliability of multiple sprint running performance indices. J Strength Cond Res. 2007;21(3):857-9.
4. Rampinini E, Sassi A, Morelli A, Mazzoni S, Fanchini M, Coutts AJ. Repeated-sprint ability in professional and amateur soccer players. Appl Physiol Nutr Metab. 2009;34(6):1048-54.

5. Comfort P, Stewart A, Bloom L, Clarkson B. Relationships between strength, sprint, and jump performance in well-trained youth soccer players. J Strength Cond Res. 2014;28(1):173-7.

6. Helgerud J, Rodas G, Kemi OJ, Hoff J. Strength and endurance in elite football players. Int J Sports Med. 2011;32(9):677-82.

7. Bangsbo J, Mohr M, Krustrup P. Physical and metabolic demands of training and match-play in the elite football player. J Sports Sci. 2006;24(7):665-74.

8. Rhea MR, Ball SD, Phillips WT, Burkett LN. A comparison of linear and daily undulating periodized programs with equated volume and intensity for strength. J Strength Cond Res. 2002;16(2):250-5.

9. Apel JM, Lacey RM, Kell RT. A comparison of traditional and weekly undulating periodized strength training programs with total volume and intensity equated. J Strength Cond Res. 2011;25(3):694-703.

10. Fleck SJ, Kraemer WJ. Designing Resistance Training Programs. 4th ed. Champaign: Human Kinetics. 2014. 520 p.

11. de Hoyo M, Gonzalo-Skok O, Sañudo B, Carrascal C, Plaza-Armas JR, Camacho-Candil F, et al. Comparative Effects of In-Season Full-Back Squat, Resisted Sprint Training, and Plyometric Training on Explosive Performance in U-19 Elite Soccer Players. J Strength Cond Res. 2016;30(2):368-77.

12. Spineti J, Figueiredo T, Oliveira VB, Assis M, Oliveira LF, Miranda $\mathrm{H}$, et al. Comparison between traditional strength training and complex contrast training on repeated sprint ability and muscle architecture in elite soccer players. J Sports Med Phys Fitness. 2016;56(11):1269-78.

13. Zourdos MC, Jo E, Khamoui A V, Lee S-R, Park B-S, Ormsbee MJ, et al. Modified Daily Undulating Periodization Model Produces Greater Performance Than a Traditional Configuration in Powerlifters. J Strength Cond Res. 2016;30(3):784-91.

14. Harries SK, Lubans DR, Callister R. Systematic Review and Meta-analysis of Linear and Undulating Periodized Resistance Training Programs on Muscular Strength. J Strength Cond Res. 2015;29(4):1113-25.

15. Alvar B, Wenner R, Dodd DJ. The Effect Of Daily Undulated Periodization As Compared To Linear Periodization In Strength Gains Of Collegiate Athletes. J Strength Cond Res. 2010;24:1.

16. Lopes CR, Crisp AH, Germano MD, Mattos RS, Sindorf MAG, Mota GR da, et al. Effects of Pre-Season Short-Term Daily Undulating Periodized Training on Muscle Strength and Sprint Performance of Under-20 Soccer Players. Int J Sci Cult Sport. 2015;3(10):64-64.

17. Hopkins WG, Marshall SW, Batterham AM, Hanin J. Progressive statistics for studies in sports medicine and exercise science. Med Sci Sports Exerc. 2009;41(1):3-13.

18. Buchheit M, Mendez-Villanueva A, Delhomel G, Brughelli M, Ahmaidi S. Improving Repeated Sprint Ability in Young Elite Soccer Players: Repeated Shuttle Sprints Vs. Explosive Strength Training. J Strength Cond Res. 2010;24(10):2715-22.

19. Tønnessen E, Shalfawi SA, Haugen T, Enoksen E. The Effect of 40-m Repeated Sprint Training on Maximum Sprinting Speed, Repeated Sprint Speed Endurance, Vertical Jump, and Aerobic Capacity in Young Elite Male Soccer Players. J Strength Cond Res. 2011;25(9):2364-70. 
20. Silva JR, Nassis GP, Rebelo A. Strength training in soccer with a specific focus on highly trained players. Sport Med. 2015;1(1):17.

21. Hill-Haas S, Bishop D, Dawson B, Goodman C, Edge J. Effects of rest interval during high-repetition resistance training on strength, aerobic fitness, and repeated-sprint ability. J Sports Sci. 2007;25(6):619-28.

22. Iaia FM, Fiorenza M, Perri E, Alberti G, Millet GP, Bangsbo J. The Effect of Two Speed Endurance Training Regimes on Performance of Soccer Players. Sandbakk O, organizador. PLoS One. 2015;10(9):e0138096.

23. Enright K, Morton J, Iga J, Drust B. The effect of concurrent training organisation in youth elite soccer players. Eur J Appl Physiol. 2015;115(11):2367-81.

24. López-Segovia M, Palao Andrés JM, González-Badillo JJ. Effect of 4 months of training on aerobic power, strength, and acceleration in two under-19 soccer teams. J Strength Cond Res. 2010;24(10):2705-14.

25. Christou M, Smilios I, Sotiropoulos K, Volaklis K, Pilianidis $\mathrm{T}$, Tokmakidis SP. Effects of resistance training on the physical capacities of adolescent soccer players. J Strength Cond Res. 2006;20(4):783-91.

\section{Corresponding author}

Dr. Charles Ricardo Lopes

Universidade Metodista de Piracicaba, UNIMEP, Campus Taquaral. Rodovia do Açúcar, km 156. Piracicaba, SP, Brazil. CEP: 13400-911

E-mail: charles_ricardo@hotmail.com

Manuscript received on July 16, 2018

Manuscript accepted on August 6, 2018

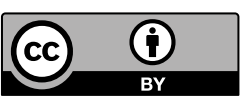

Motriz. The Journal of Physical Education. UNESP. Rio Claro, SP, Brazil - eISSN: 1980-6574 - under a license Creative Commons - Version 3.0 